\title{
Solid Phase Extraction
}

\section{Lane C. Sander}

National Institute of Standards and Technology, Gaithersburg, MD 20899, USA

lane.sander@nist.gov

Video DOI: http://doi.org/10.18434/T4DK5T

Key words: solid phase extraction; SPE; sample cleanup; extracts; method development; fractionation.

Accepted: March 6, 2017

Published: March 8, 2017

https://doi.org/10.6028/jres.122.019

\section{Summary}

Solid phase extraction is a form of liquid chromatography used in processing samples to selectively isolate constituents of interest from other compounds that may interfere with the analysis. Before a solid sample can be processed by SPE, it must first be extracted by soxhlet extraction, pressurized fluid extraction, or other method, to produce a solution that contains the analytes of interest. SPE methods usually target the analytes through selective retention mechanisms that are different from the instrumental method. The resulting processed samples are simpler than the original extracts, and may result in improved analytical results. This presentation will provide an overview of the theory and practice of solid phase extraction, and will include a demonstration of the use of different types of SPE devices. ${ }^{1}$

\footnotetext{
${ }^{1}$ Contribution of the National Institute of Standards and Technology. Not subject to copyright. Certain commercial equipment, instruments, or materials are identified to specify adequately the experimental procedure. Such identification does not imply recommendation or endorsement by the National Institute of Standards and Technology, nor does it imply that the materials or equipment identified are the best available for the purpose.
} 